\title{
Congruity influences memory and judgments of learning during survival processing
}

\author{
Christopher C. Palmore • Arturo D. Garcia • \\ L. Paige Bacon • Courtney A. Johnson • \\ William L. Kelemen
}

Published online: 19 November 2011

(C) Psychonomic Society, Inc. 2011

\begin{abstract}
A recent line of research has suggested that memory systems evolved to encode fitness-relevant information more effectively than other types of information-a phenomenon known as the "survival processing effect" (Nairne, Thompson, \& Pandeirada Journal of Experimental Psychology: Learning, Memory, and Cognition 33:263-273, 2007). However, the basis for the effect has been debated. In addition, it is unknown whether or not individuals will adjust their judgments of learning (JOLs) to reflect the survival processing effect. In three experiments, participants rated 16 words for their relevance to a survival scenario and another 16 words for their relevance to a bank robbery scenario. In Experiment $1 \mathrm{~A}$ (with no JOLs), the survival processing effect emerged; in Experiment 1B (with JOLs), no survival processing effect emerged, but JOLs were higher in the survival condition. In both cases, these findings were confounded by higher relevance ratings in the survival condition. In Experiment 2, relevance was manipulated within each list, and the survival processing effect was eliminated. Instead, both recall and JOL magnitude were related to level of congruity between the words and type of processing. Together, these results provide further evidence for the role of congruity in the survival processing effect and JOLs.
\end{abstract}

C. C. Palmore · A. D. Garcia • L. P. Bacon · C. A. Johnson Department of Psychology, California State University, Long Beach, CA 90840, USA

W. L. Kelemen $(\bowtie)$ Department of Psychology, Texas State University, 601 University Drive,

San Marcos, TX 78666, USA

e-mail: wkelemen@txstate.edu
Keywords Congruity · Judgments of learning · Metacognition · Survival processing

The mnemonic benefits of "survival processing" have been the focus of much recent memory research. In the groundbreaking study of this topic, Nairne, Thompson, and Pandeirada (2007) asked participants to rate words according to how relevant they were for surviving in the grasslands of a foreign land (e.g., securing food and providing protection from predators) as compared with control conditions in which participants rated the words according to other deep levels of processing such as selfrelevance, pleasantness, and moving to a foreign land. Their work was based on the idea that human memory systems evolved to enhance survival by giving priority to fitness-relevant information. Consistent with this idea, survival processing produced higher levels of recall than did all other conditions. In addition, the benefit generalized across several methodological adaptations such as those using different designs (i.e., within vs. between-subjects design) or types of memory tests (i.e., free recall vs. recognition tests). In a separate study, Nairne, Pandeirada, and Thompson (2008) showed that survival processing produced better memory than did additional memoryenhancing manipulations, including the use of pleasantness ratings, self-reference, imagery, and generation of items during processing. In addition, the survival advantage remains robust with pictures as stimuli instead of words (Otgaar, Smeets, \& van Bergen, 2010).

The mechanisms and boundary conditions of this powerful effect remain elusive. Burns, Hwang, and Burns (2011) proposed that the mnemonic advantage produced by survival processing derives from the combination of itemspecific processing and relational processing during encod- 
ing. Across four experiments, they examined memory for categorized lists following each type of processing (either alone or in combination) as compared with survival processing. In general, the survival processing benefit was reduced or eliminated when both types of processing were elicited on the control conditions, and it emerged when only one type of processing was elicited in the control conditions. Thus, the mechanisms behind survival processing may utilize both relational and item-specific processing, which have been shown to enhance memory in other contexts (e.g., Hunt \& McDaniel, 1993).

Another important factor that may contribute to the survival processing effect is the level of congruity between the words and processing condition. For example, Schulman (1974) showed that memory performance was facilitated when the encoding cues were congruent with target words (e.g., Is a BUBBLE a sphere?) instead of incongruent (e.g., Is a CHAPTER slippery?). The memory advantage produced by congruity also was demonstrated clearly by Craik and Tulving (1975) using a levels-ofprocessing framework. In the case of survival processing, which can be considered a "very deep" level of processing, it may be that the target words are perceived as more congruent with the concept of survival than the labels assigned to control conditions. Butler, Kang, and Roediger (2009, Experiments 2 and 3) tested this possibility by controlling the level of congruity between the target words and the type of processing (either a grasslands survival scenario or a bank robbery scenario that was matched on levels of novelty, arousal, and media exposure; see Kang, McDermott, \& Cohen, 2008). Much to their surprise, Butler et al. found no significant difference in recall between the survival condition and the robbery condition when congruity was controlled within lists (Experiments 2 and 3) in contrast with a typical uncontrolled procedure (Experiment 1). Overall, they found that words that were congruent with the processing scenario were more likely to be recalled than those incongruent with the processing scenario, regardless of the type of processing.

Butler et al.'s (2009) findings were the first published data to show a boundary condition of the survival processing effect. More recently, Nairne and Pandeirada (2011) reported the results of five experiments in a commentary on that paper. They found a survival processing advantage for lists comprising only incongruent words (Experiment 2) and congruent words (Experiment 3). When participants saw both types of words on the lists (Experiment 4), the survival processing advantage was statistically significant only for the incongruent words and a strong congruity effect emerged. Nevertheless, Nairne and Pandeirada concluded that "the null effects of survival processing obtained by Butler et al. (2009) may not generalize beyond their particular experimental design" (p. 547).
The present research further examined the role of congruity in survival processing. Nairne and Pandeirada (2011) suggested that methodological differences between their study and that of Butler et al. (2009) may account for the discrepant findings. Specifically, Butler et al. used lists that were two-thirds incongruent and one-third congruent words, whereas the closest replication reported by Nairne and Pandeirada (Experiment 4) used a balanced list (half incongruent and half congruent words). In addition, Butler et al. used longer word lists (containing 45 words), whereas Nairne and Pandeirada used lists of approximately 30 words. We tested for survival processing and congruity effects using shorter, balanced lists - precisely the conditions under which Nairne and Pandeirada failed to replicate the findings of Butler et al.

A second purpose of the present research was to test whether participants' judgments of learning (JOLs) provided during encoding would reflect the survival processing manipulation. JOLs can be provided for words during encoding, and they refer to the likelihood of future memorability. JOLs are considered to be inferential in nature and can be sensitive to a variety of factors (Koriat, 1997), including intrinsic and extrinsic cues. The former refers to properties of the items themselves that make them more or less memorable; the latter includes the type of processing during study (such as survival processing). For example, Matvey, Dunlosky, and Schwartz (2006) examined an extrinsic cue, categorical relatedness across words, and found that participants produced significantly higher immediate JOLs and higher levels of mean recall for sets of related words (e.g., peach, grape, cherry, plum) when compared with sets of unrelated words (e.g., corn, football, perch, lily). As mentioned previously, Burns et al. (2011) demonstrated the importance of categorical relatedness in recall during survival processing, obtaining no survival processing effect when the control condition elicited both relational and itemspecific processing. Thus, in the present study, the survival processing effect should be strongest in Experiment 1A, in which lists of unrelated words were used as stimuli with no JOLs. Conversely, the survival processing effect should be weakened in Experiment 2 because stimuli were selected to be highly relevant to one of two scenarios, which may have encouraged relational processing between stimuli in addition to item-specific processing.

\section{Experiments 1A and 1B}

In Experiment 1A, we examined performance using a typical survival processing procedure compared with a bank robbery scenario developed by Kang et al. (2008). We hypothesized increased recall in the survival condition as 
compared with the robbery condition using these standard (no JOL) procedures. In Experiment 1B, we explored whether the survival processing benefit would extend to participants who provided JOLs, and if so, whether JOLs would be sensitive to the survival processing effect.

\section{Method}

Participants Sixty undergraduate students (38 women, 22 men) enrolled in an introductory psychology course at a large urban university volunteered to participate in exchange for course credit. The age of participants ranged from 18-22 years old $(M=18.88 ; S D=1.11)$. Participants were recruited using the Psychology Department Subject Pool and were treated in accordance with the ethical principles of the American Psychological Association.

Materials The stimuli consisted of two lists of 16 words each that were used in Experiments 1 and 2 of Nairne et al.'s (2007) study. The survival instructions were taken from Nairne et al., and the robbery instructions were taken from Kang et al. (2008) as follows:

Survival In this task, we would like you to imagine that you are stranded in the grasslands of a foreign land, without any basic survival materials. Over the next few months, you'll need to find steady supplies of food and water and protect yourself from predators. We are going to show you a list of words, and we would like you to rate how relevant each of these words would be for you in this survival situation. Some of the words may be relevant and others may not—it's up to you to decide.

Robbery In this task, we would like you to imagine that you are leading a heist of a well-guarded bank. Over the next few months, you'll need to find people to help you, make a plan, and gather any supplies you might need. We are going to show you a list of words, and we would like you to rate how relevant each of these words would be for you in this robbery situation. Some of the words may be relevant and others may not - it's up to you to decide.

In Experiment 1B, additional instructions were included: "In addition to its relevance, please rate how likely you are to remember each word on a subsequent memory test. Rate the probability of recall on a percentage scale (where $0 \%$ means no chance of recall and $100 \%$ means definite recall)."

The instructions and stimuli were presented using a standard LCD projector. The timing was controlled using iMovie 09 software. Participants provided all responses using paper and pencil. They rated the relevance of each item using a 5-point Likert scale, in which a rating of 1 indicated that the word was not relevant at all to the scenario and a rating of 5 indicated that the word was extremely relevant. In Experiment 1B, JOLs ranged from $0 \%$ to $100 \%$ in increments of $20 \%$. In addition, participants in Experiment $1 \mathrm{~B}$ provided an aggregate JOL (ranging from $0-16)$ at the end of each list.

Design and Procedure In both Experiments 1A and 1B, type of processing was a within-subjects manipulation. Thirty participants completed Experiment 1A, and a separate sample of 30 participants completed Experiment 1B. Of the 30 participants in each experiment, 15 completed the survival processing manipulation first, and 15 completed the robbery processing condition first. The order of stimuli was randomized before the experiments and was held constant across all participants.

Participants were tested in small groups ranging from 15 people in a single session that lasted approximately 45 min. Upon arrival, they were seated at a table and were asked to provide informed consent. Then, the instructions were provided. Two practice items were provided prior to beginning each processing condition, which were not scored. No participants had any questions following the practice items. All stimuli were presented one at a time in a central location on the screen for $7 \mathrm{~s}$ each. During presentation, all participants were asked to rate the relevance of each item; participants in Experiment 1B additionally provided an immediate JOL during this time as well as an aggregate JOL at the end of all 16 items in each processing condition.

Following the rating task, all participants completed six 3digit multiplication problems that were presented one at a time in a central location on the screen for $20 \mathrm{~s}$ each. Afterward, participants were given a blank sheet of paper and were asked to write down as many words as they could remember from both of the earlier lists. The free recall task lasted $10 \mathrm{~min}$, and participants were informed that the order in which they recalled the items was unimportant. At the end of the experimental session, each participant completed a demographic questionnaire, was debriefed, and then dismissed.

\section{Results and discussion}

All statistical analyses were conducted with $\alpha=.05$ using the PASW 18.0 statistics program. Effect sizes were derived using an online calculator at http://www.cognitiveflexibility. org/effectsize. For Cohen's $d$, this calculator uses a correction for the dependence of means derived from Morris and DeShon's (2002) Equation 8.

Recall One major prediction was that mean levels of recall would be higher in the survival condition than in 
the robbery condition. As expected, recall in Experiment $1 \mathrm{~A}$ was significantly greater in the survival condition $(M=.57, S D=.18)$ than in the robbery condition $(M=.49$, $S D=.19), t(29)=1.71$, standard error of the difference $(S E D)=.05, d=.31$. These mean levels of recall are nearly identical to those reported in Experiment 1 of Butler et al. $(2009 ; M \mathrm{~s}=.57$ and .50 , respectively), and they represent the standard survival processing effect and procedures.

In contrast, no survival processing benefit emerged in Experiment 1B: Recall in the survival condition $(M=.47$, $S D=.15$ ) was only slightly (and nonsignificantly) higher than the robbery condition $(M=.45, S D=.19), t(29)=$ $0.47, S E D=.05$. Thus, the survival processing effect observed did not extend to participants who provided JOLs. Several methodological changes in this condition may be involved. First, the JOL task necessarily involved intentional learning: Participants were warned to expect an upcoming test and to provide JOLs accordingly. In contrast, nearly all previous studies of survival processing have used incidental learning (one unpublished poster presentation by Ceo \& Nairne (2007) reported an intentional learning condition and found no advantage for survivalrelated words as compared with non-survival words). In addition, participants in Experiment 1B provided two ratings (i.e., relevance and JOLs) during the 7-s interval; participants in Experiment 1A provided only one rating (i.e., relevance). We opted to hold the retention interval constant between Experiments $1 \mathrm{~A}$ and $1 \mathrm{~B}$, but it is possible that requiring both ratings in the latter case weakened the survival processing effect. A third possibility is that eliciting JOLs somehow increased relational processing between items. If both relational and item-specific processing are elicited in a control condition, then the relative survival processing advantage can be reduced (Burns et al., 2011). This third possibility is speculative because both Burns et al. and Matvey et al. (2006) manipulated the relatedness of items within lists, and we did not. In Experiment 2, however, we did use words highly related to one of two processing conditions, resulting in categorized lists.

Judgments of learning Participants in Experiment 1B made both item-by-item and aggregate JOLs. Data for both types of JOLs were converted to proportions for ease of comparison with recall scores. Participants' item-by-item JOLs were significantly higher in the survival condition $(M=.49, S D=.15)$ than in the robbery condition $(M=$ $.42, S D=.16), t(29)=2.55, S E D=.03, d=.46$. Thus, participants expected their recall to be higher in the survival condition than in the robbery condition, even though the actual recall benefit was not significant in Experiment 1B. No significant difference in aggregate JOLs was observed between processing conditions $\left(M_{\text {survival }}=.48, S D_{\text {survival }}=.18\right.$ vs. $M_{\text {robbery }}=.47$,
$\left.S D_{\text {robbery }}=.17\right)$. Thus, the increased confidence for items in the survival condition was limited to the item-by-item JOLs and did not extend to global confidence for the list.

Relevance ratings Participants rated the relevance of each item to their assigned type of processing for each list. In both Experiments $1 \mathrm{~A}$ and $1 \mathrm{~B}$, relevance ratings were significantly higher in the survival condition $(M=2.73$, $S D=.61$, and $M=2.62, S D=.67$, respectively) than in the robbery condition $(M=2.17, S D=.52$, and $M=2.25, S D=$ .58 , respectively), $t(29)=4.83, S E D=.12, d=.89$, for Experiment $1 \mathrm{~A}$, and $t(29)=4.28, S E D=.09, d=.79$, for Experiment 1B. These findings muddy the interpretation of the survival processing differences observed for recall and JOLs. Instead of a survival processing advantage per se, the differences may reflect a congruity effect between the task and items (cf., Experiment 3 of Nairne et al., 2007).

To test this possibility, we examined the relationship between congruity and recall. The mean Gamma correlation $(G)$ between rated relevance and recall for each participant (collapsed across both experiments) was .21 $(S D=.43)$ in the survival condition and $.34(S D=.40)$ in the robbery condition. Both mean values were significantly nonzero using one-sample $t$ tests, $t(59)=3.75$, and $t(59)=6.73$, respectively. Thus, rated relevance was directly related to future memory performance. The magnitude of this relationship did not differ significantly between type of processing conditions, $t(59)=1.72, p=.09$. The relationship between rated relevance and JOL magnitude was even stronger: $G$ was $.72(S D=.32)$ in the survival condition and $.59(S D=.47)$ in the robbery condition. Again, both mean values were significantly nonzero, $t(29)=12.18$ in the survival condition and $t(29)=6.83$, in the robbery condition, and again, the difference between survival vs. robbery conditions was not significant, $t(29)=1.54, p=.14$. Overall, congruity was directly related to both recall and JOL magnitude within each type of processing; thus, congruity may have contributed to the differences between processing conditions.

\section{Experiment 2}

To further explore the role of congruity in recall and JOL magnitude, we adopted the methodology of Butler et al. (2009, Experiments 2 and 3). Specifically, we balanced the congruity of words with the type of processing elicited in each list, whereas in Experiments 1A and 1B (and in most previous studies of survival processing), the congruity of the items was not controlled but merely assessed post hoc. By including highly congruent and highly incongruent words on each list, the relative contributions of processing 
type and congruity to recall and JOL magnitude could be assessed. Thus, it was possible to determine whether performance would vary according to congruity within a word list, the type of processing between word lists, or both.

\section{Method}

Participants A sample of 58 students (40 women, 18 men) who did not participate previously was recruited as before. The age of participants ranged from 18-25 years old $(M=19.40 ; S D=1.49)$. All data from two additional participants were discarded because it was impossible to decipher several of their responses.

Materials Two lists of 16 words each were constructed so that half of the words on each list were closely related to survival and the other half of the words were closely related to a bank robbery. The order of the words on each list was randomized before the study began and remained constant across all participants. The 15 robbery words and 15 survival words in the Appendix of Butler et al. (2009) were utilized; two additional words were added (flint for the survival condition and money for the bank robbery condition) to generate 32 total words, as in Experiments $1 \mathrm{~A}$ and $1 \mathrm{~B}$.

Design and procedure The processing condition (survival vs. robbery) varied within subjects, and the JOL group (JOLs vs. no JOLs) varied between subjects. In addition, the congruity of each item now varied within subjects: Eight of the words on each list were highly relevant to survival, and the other eight words were highly relevant to a bank robbery. The instructions and procedures were identical to those in Experiments 1A and 1B.

\section{Results and discussion}

Recall Mean levels of recall across conditions are listed at the top of Table 1. Perhaps most notable is the complete lack of a survival processing advantage. Separate $2 \times 2$ (processing type $\times$ congruity) within-subjects ANOVAs in the JOL and no-JOL groups revealed no main effects of processing type $\left(F_{\mathrm{s}}<0.65 ; p \mathrm{~s}>.42\right)$. However, congruity did produce a statistically significant main effect, $F(1,28)=33.95, M S E=.03, \eta^{2}$ partial $=.55$, in the JOL group, and a moderate effect size but nonsignificant main effect in the no-JOL group, $F(1,28)=2.68$, $M S E=.035, \eta_{\text {partial }}^{2}=.09$. No interaction terms were statistically significant. These findings are consistent with the observation of Butler et al. (2009) that the survival processing effect can be eliminated when congruity is
Table 1 Mean levels of recall, JOL magnitude, and relevance ratings as a function of JOL group, congruity, and processing type in Experiment 2

\begin{tabular}{|c|c|c|c|c|}
\hline & \multicolumn{2}{|c|}{ No JOLs Provided } & \multicolumn{2}{|c|}{ JOLs Provided } \\
\hline & $\begin{array}{l}\text { Congruent } \\
\text { Words }\end{array}$ & $\begin{array}{l}\text { Incongruent } \\
\text { Words }\end{array}$ & $\begin{array}{l}\text { Congruent } \\
\text { Words }\end{array}$ & $\begin{array}{l}\text { Incongruent } \\
\text { Words }\end{array}$ \\
\hline Recall & $M S D$ & $M S D$ & $M S D$ & $M S D$ \\
\hline Survival & .56 .19 & .49 .24 & .62 .21 & .49 .13 \\
\hline Robbery & .57 .22 & .52 .20 & .65 .18 & .42 .19 \\
\hline \multicolumn{5}{|c|}{ JOL Ratings } \\
\hline Survival & NA & NA & .77 .13 & .33 .17 \\
\hline Robbery & NA & NA & .79 .12 & .37 .22 \\
\hline \multicolumn{5}{|c|}{ Relevance Ratings } \\
\hline Survival & 4.34 .47 & 2.14 .73 & 4.31 .52 & 1.96 .72 \\
\hline Robbery & 4.53 .39 & 1.98 .60 & 4.40 .42 & 1.92 .52 \\
\hline
\end{tabular}

NA indicates "not applicable" because no JOL ratings were collected in that group

tightly controlled by the experimenter. In fact, congruity played a larger role in determining recall performance than did the type of processing in this experiment.

Judgments of learning Mean item-by-item JOLs by condition are listed in middle of Table 1 . We conducted another 2 $\mathrm{X} 2$ ANOVA on JOL magnitude and found a pattern similar to the recall data: Congruity produced a significant main effect, $F(1,28)=181.55, M S E=1.73, \eta_{\text {partial }}^{2}=.87$, whereas neither processing type nor the interaction term was statistically significant $\left(F_{\mathrm{S}}<1.26 ; p \mathrm{~s}>.27\right)$. These results mirror the recall data in that participants were more confident in their memory when the item was congruent with the type of processing elicited, regardless of whether the survival or robbery scenario was being used. As before, no significant difference in aggregate JOLs was observed between conditions $\left(M_{\text {survival }}=.54, S D_{\text {survival }}=.16\right.$ vs. $\left.M_{\text {robbery }}=.54, S D_{\text {robbery }}=.17\right)$.

Relevance Ratings Mean relevance ratings by condition are listed at the bottom of Table 1 . The items selected to be more congruent for each type of processing were in fact rated as more relevant by the participants. The $2 \times 2$ ANOVAs confirmed a large and statistically significant effect of congruity on relevance ratings, $F(1,28)=$ $587.73, M S E=.20, \eta^{2}$ partial $=.96$, for the JOL group, and $F(1,28)=493.07, M S E=.17, \eta_{\text {partial }}^{2}=.95$, for the no-JOL group. In contrast with Experiments $1 \mathrm{~A}$ and 1B, relevance ratings did not vary according to processing type in either case $(F \mathrm{~s}<0.09 ; p \mathrm{~s}>.77)$, suggesting that our effort to control relevance across processing conditions was successful. 
Finally, we examined the relationship between rated relevance and both recall and JOLs. For recall, $G$ was .31 $(S D=.38)$ in the survival condition and $.22(S D=.39)$ in the robbery condition. Both mean values were significantly nonzero using one-sample $t$ tests, $t(56)=6.24$, and $t(56)=$ 4.34 , respectively, and the difference did not vary between groups $t(55)=1.38, p=.18$. The relationship was even stronger for JOLs: $G$ was $.91(S D=.13)$ in the survival condition and $.87(S D=.25)$ in the robbery condition. Again, both mean values were significantly nonzero, $t(28)=37.65$, and $t(28)=18.56$, respectively, and the difference did not vary between groups, $t(28)=1.29, p=.21$. Thus, congruity was related to both recall and JOL magnitude within each type of processing, replicating the pattern in Experiments 1A and $1 \mathrm{~B}$.

\section{General discussion}

Three experiments were conducted to examine the role of congruity in survival processing and to determine whether the survival processing effect would generalize to metacognitive judgments. In Experiment $1 \mathrm{~A}$, the standard survival processing effect emerged. In Experiment 1B, however, only JOLs were higher in the survival condition as compared with a robbery condition. In both cases, the level of congruity between the words and conditions also varied, which presented an interpretative challenge. We manipulated level of congruity in Experiment 2, and it produced larger effects on both recall and JOLs than on type of processing. These data support the findings of Butler et al. (2009) and provide further evidence that the survival processing effect can be reduced or eliminated using conditions in which the word lists are balanced with respect to congruity. Our data do not support the claim that the congruity effects noted by Butler et al. may have been artifacts of their experimental design. In fact, the role of congruity has been manipulated in only one other experiment (Experiment 4 of Nairne \& Pandeirada, 2011), and the survival processing effect was reduced in magnitude.

Congruity may be an important factor in understanding the proximate mechanisms of the survival processing effect; however, it clearly is only a partial explanation of a robust phenomenon. For example, in Nairne et al.'s (2007) seminal study, they observed substantial survival advantages for recall when mean relevance ratings were nearly identical between conditions (Experiment 1), marginally higher in the survival condition ( $p=.08$ in Experiment 2$)$, significantly higher for the survival condition (Experiment 3 ), and significantly lower in the survival condition
(Experiment 4). Nevertheless, Nairne et al. did report in Experiment 1 (when mean levels of relevance were nearly identical between conditions) that recall increased in conjunction with the relevance of the items in both nonsurvival and survival processing conditions. Our $G$ correlations showed the same relationship for recall as well as JOL magnitude. Thus, congruity effects can be large in this paradigm, and they can emerge in the presence or absence of a statistically significant survival processing effect.

The JOL results also provide indirect support for the role of congruity in survival processing. First, JOL magnitude was related to congruity, as shown by the $G$ correlations. These results are consistent with findings by Matvey et al. (2006) that JOLs can be influenced by the relatedness between words in a free recall task. Perhaps more notably, the magnitude of JOLs in our Experiment 2 varied according to congruity but not according to processing type. Thus, both memory and metacognitive judgments appeared to be more sensitive to congruity than to survival processing in Experiment 2. JOLs can be sensitive to a variety of cues, including extrinsic factors such as the processing type or congruity during encoding (Koriat, 1997). Theoretically, JOLs could have been sensitive to either type of cue. Although extrinsic cues generally are not powerful determinants of JOL magnitude, they can influence JOLs, especially when they are manipulated within subjects (e.g., Carroll \& Nelson, 1993). In fact, congruity was manipulated within subjects in the present study, Experiments 2 and 3 of Butler et al., and Experiment 4 of Nairne and Pandeirada (2011), and the survival processing effect was reduced or eliminated in all cases. When congruity was merely controlled in other studies (e.g., Nairne and Pandeirada's (2011) Experiments 2 and 3 ), then the survival processing effect tended to be larger.

To extend these findings, it would be informative to use materials other than the survival and bank robbery lists as well as to directly compare both between- and withinsubjects manipulations of congruity. Overall, the present data support the idea that level of congruity may reflect an important boundary condition of this phenomenon (Butler et al. 2009). Another boundary condition may involve the type of processing (item-specific vs. relational) elicited by control conditions (Burns et al., 2011). Thus, evidence is accumulating that an evolution-based module may not be the sole explanation for the survival processing effect. Instead, it seems likely the ultimate theoretical account will be multifactorial.

Author Note Portions of these data were presented in April 2011 at the $91^{\text {st }}$ Annual Convention of the Western Psychological Association in Los Angeles. 


\section{References}

Butler, A. C., Kang, S. H., \& Roediger, H. L., III. (2009). Congruity effects between materials and processing tasks in the survival processing paradigm. Journal of Experimental Psychology: Learning, Memory, and Cognition, 35, 1477-1486. doi:10.1037/a0017024

Burns, D. J., Hwang, A. J., \& Burns, S. A. (2011). Adaptive memory: Determining the proximate mechanisms responsible for the memorial advantages of survival processing. Journal of Experimental Psychology: Learning, Memory, and Cognition, 37, 206-218. doi:10.1037/a0021325

Carroll, M., \& Nelson, T. O. (1993). Effect of overlearning on the feeling of knowing is more detectable in within-subject than in between-subject designs. The American Journal of Psychology, 106, 227-235. doi:10.2307/1423169

Ceo, D.A., \& Nairne, J.S. (2007, November). Adaptive memory: Does survival value matter? Poster session presented at the $48^{\text {th }}$ Annual Meeting of the Psychonomic Society, Long Beach, CA.

Craik, F. I. M., \& Tulving, E. (1975). Depth of processing and retention of words in episodic memory. Journal of Experimental Psychology: General, 104, 268-294. doi:10.1037/0096-3445.104.3.268

Hunt, R. R., \& McDaniel, M. A. (1993). The enigma of organisation and distinctiveness. Journal of Memory and Language, 32, 421445. doi:10.1006/jmla.1993.1023

Kang, S. H. K., McDermott, K. B., \& Cohen, S. M. (2008). The mnemonic advantage of processing fitness-relevant information. Memory \& Cognition, 36, 1151-1156. doi:10.3758/MC.36.6.1151
Koriat, A. (1997). Monitoring one's own knowledge during study: A cue-utilization approach to judgments of learning. Journal of Experimental Psychology: General, 126, 349-370. doi:10.1037/ 0096-3445.126.4.349

Matvey, G. M., Dunlosky, J., \& Schwartz, B. L. (2006). The effects of categorical relatedness on judgments of learning (JOLs). Memory, 14, 253-261. doi:10.1080/09658210500216844

Morris, S. B., \& DeShon, R. P. (2002). Combining effect size estimates in meta-analysis with repeated measures and independent-groups designs. Psychological Methods, 7, 105-125. doi:10.1037/1082-989X.7.1.105

Nairne, J. S., Thompson, S. R., \& Pandeirada, J. N. S. (2007). Adaptive memory: Survival processing enhances retention. Journal of Experimental Psychology: Learning, Memory, and Cognition, 33, 263-273. doi:10.1037/0278-7393.33.2.263

Nairne, J. S., \& Pandeirada, J. N. S. (2011). Congruity effects in the survival processing paradigm. Journal of Experimental Psychology: Learning, Memory, and Cognition, 37, 539-549. doi: $10.1037 / \mathrm{a} 0021960$

Nairne, J. S., Pandeirada, J. N. S., \& Thompson, S. R. (2008). Adaptive memory: The comparative value of survival processing. Psychological Science, 19, 176-180. doi:10.111/j.14679280.2008.02064.x

Otgaar, H., Smeets, T., \& van Bergen, S. (2010). Picturing survival memories: Enhanced memory after fitness-relevant processing occurs for verbal and visual stimuli. Memory \& Cognition, 38, 23-28. doi:10.3758/MC.38.1.23

Schulman, A. I. (1974). Memory for words recently classified. Memory \& Cognition, 2, 47-52. 